\title{
DÚVIDAS DOS PACIENTES EM PÓS-OPERATÓRIO DE REVASCULARIZAÇÃO DO MIOCÁRDIO*
}

\author{
Paula Fernanda Pereira de Almeida ${ }^{1}$, Roberto Góes Júnior² ${ }^{2}$ Renata Cristina Gasparino ${ }^{3}$
}

RESUMO: Ao receber alta hospitalar, o paciente submetido a cirurgia cardíaca pode apresentar dúvidas que geram ansiedade e insegurança, por estar longe da vigilância constante da equipe de saúde. Por essa razão, o objetivo deste estudo foi identificar as dúvidas dos pacientes em pós-operatório de revascularização do miocárdio, relacionadas ao período de reabilitação. Trata-se de um estudo qualitativo e, para sua realização, foram utilizados uma ficha de caracterização sociodemográfica e um roteiro de entrevista semiestruturada. A amostra foi composta por dez pacientes e as dúvidas mais frequentes foram aquelas relacionadas a re-educação alimentar, prática de exercícios físicos, retorno ao trabalho, sintomas que podem apresentar após a alta, prática de atividades diárias, dificuldades com as incisões cirúrgicas, prática de atividade sexual, consumo de bebida alcoólica e uso de medicações. Destaca-se a importância de o enfermeiro conhecer as dúvidas dos pacientes, para que possa desenvolver intervenções individualizadas com foco na prevenção de complicações. PALAVRAS-CHAVE: Revascularização miocárdica; Período pós-operatório; Cuidados de enfermagem.

\section{QUESTIONS FROM PATIENTS IN POSTOPERATIVE PERIOD OF MYOCARDIAL REVASCULARIZATION}

\begin{abstract}
When patients undergoing cardiac surgery are discharged, they may have questions that cause anxiety and insecurity because they will be away from the constant surveillance of the health team. Therefore, the aim of the present study was to identify the questions patients in the postoperative period of myocardial revascularization had concerning the rehabilitation period. To perform this qualitative study, we have used sociodemographic characterization charts and semi-structured interviews. The sample was formed by ten patients and the most frequent questions were those concerning dietary re-education, physical exercises practice, returning to work, symptoms that can be present after discharge, common daily activities, problems with surgical incision, sexual activity, alcohol use, and medication use. Thus, nurses should know patients' questions so that individualized interventions can be developed focusing on preventing complications. KEYWORDS: Myocardial revascularization; Postoperative period; Nursing care.
\end{abstract}

\section{DUDAS DE LOS PACIENTES EN POSTOPERATORIO DE REVASCULARIZACIÓN DEL MIOCARDIO}

RESUMEN: Al ser dados de alta del hospital, el paciente sometido a una cirugía cardíaca puede presentar dudas que generan ansiedad e inseguridad, por estar lejos de la vigilancia constante del equipo de salud. Por esta razón, el objetivo de este estudio fue identificar las dudas de los pacientes en postoperatorio de revascularización del miocardio, relacionadas con el período de rehabilitación. Se trata de un estudio cualitativo, y para su realización, fueron utilizados una ficha de caracterización sociodemográfica y un guión de entrevista semiestructurada. La muestra estaba compuesta por diez pacientes y las dudas más frecuentes eran aquellas relacionadas con la a la reeducación alimentaria, práctica de ejercicios físicos, la vuelta al trabajo, síntomas que pueden presentar después del alta, la práctica de actividades cotidianas, dificultades con las incisiones quirúrgicas, práctica de actividad sexual, consumo de bebida alcohólica y uso de los medicamentos. Sobresale la importancia de que el enfermero conozca las dudas de los pacientes, para que pueda desarrollar intervenciones individualizadas con foco en la prevención de complicaciones.

PALABRAS CLAVE: Revascularización miocárdica; Periodo postoperatorio; Cuidados de enfermería.

\footnotetext{
*Artigo extraído do Trabalho de Conclusão de Curso apresentado ao Curso Superior de Enfermagem da Faculdade de Medicina de Jundiaí-FMJ para obtenção do título de bacharel em enfermagem.

${ }^{1}$ Enfermeira da Unidade Coronariana do Hospital Paulo Sacramento. Pós-graduanda em enfermagem em cardiologia pela Universidade Federal de São Paulo-UNIFESP.

${ }^{2}$ Enfermeiro da Unidade de Terapia Intensiva do Hospital Paulo Sacramento.

${ }^{3}$ Mestre em Enfermagem pela Universidade Estadual de Campinas-Unicamp.Professora Assistente do Departamento de Enfermagem da FMJ.
}

Autor correspondente:

Renata Cristina Gasparino

Faculdade de Medicina de Jundiaí

Rua Saldanha Marinho, 387 - 13215-290 - Jundiaí-SP, Brasil

Recebido: 15/08/09

E-mail: regasparino@yahoo.com.br

Aprovado: 22/10/09 


\section{INTRODUÇÃO}

As doenças cardiovasculares-DCV constituem uma importante problemática no que diz respeito à saúde da população, pois se destacam como a principal causa de morte no Brasil ${ }^{(1)}$. Considerando as altas taxas de morbimortalidade e os elevados custos implicados no diagnóstico e tratamento e que as DCV constituem um grupo de doenças multifatoriais, conhecer os seus fatores de risco contribui para que se invista em medidas que previnam os seus agravos.

Os pacientes precisam conhecer os fatores de risco das DCV, classificados como irreversíveis (idade, sexo, raça e história familiar de doença coronária) e reversíveis (hipertensão arterial, dislipidemia, tabagismo, diabetes, obesidade, sedentarismo, uso de anticoncepcionais hormonais, estresse) para que possam modificar seu estilo de vida com o intuito de minimizar o desenvolvimento dessas doenças, prevenir recorrências e melhorar sua qualidade de $\operatorname{vida}^{(2)}$.

Os fatores de risco são capazes de lesar o endotélio vascular aumentando a probabilidade do desenvolvimento da aterosclerose, uma doença crônica que leva à formação do ateroma, que pode se tornar frágil e romper. $\mathrm{O}$ ateroma roto expõe a matriz subendotelial, levando à ativação das plaquetas, geração de trombina e formação de trombo, podendo ocasionar oclusão parcial ou total do vaso, causando isquemia ou necrose tecidual ${ }^{(3)}$. A doença da artéria coronária-DAC pode ser tratada de forma clínica ou cirúrgica $^{(4)}$.

A cirurgia cardíaca, por se tratar de um procedimento complexo, traz consigo um significado especial. Os pacientes que necessitam ser submetidos a esse procedimento se sentem com baixa autoestima, ansiosos, angustiados ${ }^{(1)}$ e, muitas vezes, esses sentimentos estão relacionados à falta de conhecimento da própria doença, procedimento e recuperação.

Frente à reabilitação após a realização de uma cirurgia cardíaca, algumas complicações podem ser observadas. Quando um paciente submetido a esse procedimento recebe alta hospitalar, ele pode se sentir ansioso e desprotegido por estar longe da vigilância constante da equipe de saúde e esses sentimentos podem influenciar negativamente na sua recuperação $0^{(5)}$.

Portanto, o objetivo desse estudo foi identificar as dúvidas dos pacientes em pós-operatório de cirurgia de revascularização do miocárdio-RVM, considerando que esses dados podem subsidiar intervenções de enfermagem mais adequadas e individualizadas, melhorar a qualidade da assistência oferecida, influenciar positivamente a recuperação desses pacientes e diminuir os riscos de complicações decorrentes do processo de reabilitação.

\section{MÉTODO}

Trata-se de um estudo exploratório, descritivo, transversal e com abordagem qualitativa que foi desenvolvido em um hospital geral do tipo filantrópico no interior do Estado de São Paulo, que conta com cerca de 220 leitos e realiza em média 24 mil atendimentos por mês, sendo 99\% destes por intermédio do SUS.

A coleta de dados foi realizada entre o período de agosto a outubro de 2008. A amostra, selecionada por conveniência, foi constituída por dez sujeitos que foram submetidos à cirurgia de RVM. Adotou-se o critério de saturação dos dados para delimitar o tamanho da mesma que consiste em encerrar a coleta quando as convergências das falas dos sujeitos apresentarem repetições ${ }^{(6)}$.

O projeto obteve aprovação do Comitê de Ética em Pesquisa da Faculdade de Medicina de Jundiaí e da instituição cenário do estudo.

Os critérios de inclusão na amostra foram: ter sido submetido à cirurgia de RVM; ter idade igual ou superior a 18 anos; ser capaz de comunicar-se verbalmente e compreender a finalidade do estudo; encontrar-se na unidade de internação, ou seja, já ter recebido alta da unidade de terapia intensiva; e concordar em participar do estudo e assinar o Termo de Consentimento Livre e Esclarecido.

Para coleta de dados foram utilizados dois instrumentos: ficha de caracterização sociodemográfica e roteiro de entrevista semiestruturada por meio da questão norteadora: Pense nas atividades que o Sr.(a) realizava antes da cirurgia e em tudo o que fazia parte da sua rotina. Pense também em tudo o que mudará quando o Sr.(a) sair do hospital e for para casa. Quais são suas dúvidas quanto à sua recuperação?

Quando os pacientes não expressaram dúvidas na resposta a essa primeira questão, os pesquisadores elaboraram as questões subsequentes com base em complicações apresentadas pelos pacientes em outros estudos $^{(1,7-10)}$.

Os sujeitos foram abordados pelos pesquisadores em horários convenientes a ambos. As entrevistas foram gravadas, após permissão dos pacientes e transcritas na íntegra, respeitando os critérios de originalidade dos discursos. Como critérios de seleção dos depoimentos 
para exposição e posterior discussão, foi adotado o conceito de expressões-chave, que consiste em trechos ou transcrições literais dos discursos que revelam a essência e conteúdo do depoimento(11).

\section{RESULTADOS}

A amostra foi composta por dez participantes, com uma média de idade de 56 anos $(\mathrm{dp} \pm 6,4)$ e tempo médio de pós-operatório de quatro dias ( $\mathrm{dp} \pm 0,82)$. A maioria era do sexo masculino (80\%), casado (80\%), com vida sexual ativa (90\%), possuía ensino fundamental incompleto (50\%) e exercia trabalho remunerado (70\%).

Os fatores de risco para DCV, presentes na amostra, estão representados na Tabela 1.

Tabela 1 - Distribuição de frequências e porcentagens dos fatores de risco presentes nos pacientes em pós-operatório de RVM. Jundiaí, 2008

\begin{tabular}{lcc}
\hline Variáveis & \multicolumn{2}{c}{ Distribuição dos pacientes } \\
\cline { 2 - 3 } & n. & $\%$ \\
\hline Fatores de risco & & \\
Dieta desequilibrada & 10 & $100 \%$ \\
Idade & 10 & $100 \%$ \\
Estresse & 8 & $80 \%$ \\
História familiar & 7 & $70 \%$ \\
Dislipidemia & 6 & $60 \%$ \\
Diabetes Mellitus & 6 & $60 \%$ \\
Sedentarismo & 6 & $60 \%$ \\
Tabagismo & 6 & $60 \%$ \\
Hipertensão & 5 & $50 \%$ \\
Uso de hormônios & 2 & $20 \%$ \\
Excesso de peso & 1 & $10 \%$ \\
Consumo de bebida alcoólica & 1 & $10 \%$ \\
\hline
\end{tabular}

Analisando as respostas dos sujeitos, observouse que $80 \%$ dos entrevistados manifestaram dúvidas em mais da metade dos temas abordados.

Quando questionados quanto aos hábitos alimentares, nove sujeitos (90\%) alegaram possuir dúvidas relacionadas ao tempo de duração da dieta e possibilidade de abrir exceções:

[...] vou seguir a dieta que o médico me passou que é fervorosa! [...] Pretendo seguir daqui prá frente, mas será que vou ter que seguir pro resto da vida? (P.8).
[...] assim, uma carninha gorda [...] uma vez por semana ou uma vez por mês acho que não tem nada a ver, né? (P.2).

Em relação ao tema exercícios físicos, nove sujeitos (90\%) alegaram possuir dúvidas relacionadas ao retorno à atividade física, tipo de exercício, frequência, intensidade e duração do mesmo, possibilidade de retorno à prática de futebol e recorrência de sintomatologia de intolerância à atividade física:

[...] olha eu sempre fui do tipo relaxado com isso, muito dificilmente eu gostava de jogar uma bolinha com uns amigos, mas já faz tempo! E agora eu vou ter que fazer? Porque eu já ouvi dizendo por aí que tem que fazer pelo menos uma caminhada, certo? Se realmente eu tiver que fazer, o que fazer e quantas vezes por semana? (P.10).

Dentre os participantes, oito (80\%) referiram dúvidas no que se refere ao retorno ao trabalho como realização de esforço físico e limitações, tempo de afastamento, possibilidade de retorno e necessidade de entrada no processo de aposentadoria:

[...] como vai ser minha vida depois da cirurgia sobre algum tipo de trabalho um pouquinho mais esforçado, eu vou ter condições de fazer esse tipo? Por exemplo, seria assim, eu vou poder ter um trabalho que eu vou poder chegar e poder levantar $25 \mathrm{~kg}, 20 \mathrm{~kg}$ e tal? (P.1).

No que se refere aos sintomas aos quais deveriam se atentar após a alta, oito (80\%) sujeitos manifestaram dúvidas quanto à recidiva dos sintomas e recorrência de infarto, quanto aos sintomas aos quais deveriam se atentar e qual conduta deveria ser tomada mediante a persistência dos sintomas do pós-operatório:

[...] eu vou poder respirar normal? [...] Tossir normal, por exemplo? Porque sabe que depois da cirurgia se der uma tosse, arrebenta tudo por dentro mesmo, dói! [...] (P.1).

[...] como minha veia estava "seca" me dava aquele "aperto", agora como trocou eu acredito que não vou mais sentir aquilo, certo? (P.7).

A grande maioria dos participantes (80\%) manifestou dúvidas em relação ao retorno às atividades 
diárias, sendo que 100\% destas basearam-se em atividades envolvendo esforços físicos:

[...] vou poder dirigir e ir prá cima e prá baixo? Vou poder carregar minha neta de cinco meses que, meu Deus, desde o mês sete que eu só cheiro, entendeu? Vou poder andar de ônibus? Porque isso é uma coisa que faz bastante esforço, o ônibus vive lotado, você fica um tempão esperando de pé até o ônibus chegar e vai espremido [...]. Na minha casa tem escadas, eu vou poder subir e descer? [...] a minha rotina era: eu acordava às cinco horas da manhã e limpava minha casa, passava roupa, lavava, aí eu tomava meu banho e ia trabalhar, entendeu? [...] Então pensando nisso, será que vou poder voltar a toda essa minha rotina, vou poder fazer meus serviços domésticos? (P.9).

No momento da entrevista, $60 \%$ dos participantes referiram dúvidas relacionadas às incisões cirúrgicas, como cuidados de higiene e realização de curativo, tempo de cicatrização, permanência da cicatriz e local onde será realizado o curativo:

[...] eu também não sei como cuidar não, o que eu tenho que usar e o que tenho que passar? Quanto tempo vai demorar prá cicatrizar? (P. 8).

Quanto às atividades sexuais, quatro (40\%) sujeitos referiram dúvidas com relação à possibilidade de sofrer prejuízos e limitações, tempo para retorno às atividades e uso de anticoncepcionais hormonais:

[...] muitas, é claro! Eu vou poder voltar à atividade normal? Porque eu tenho só cinquenta anos e eu quero viver! Quando posso fazer? Vou ter restrições, vai ter que ser sempre aquela coisa básica tipo arroz-feijão? Vou poder continuar a tomar pílulas, porque eu já tomo há muitos anos e foi o único método que eu me adaptei e quando eu tive o infarto foi a primeira coisa que o médico aboliu da minha vida (P.9).

Todos os participantes que tinham tabagismo como fator de risco para DAC afirmaram que abandonariam esse hábito e, portanto, nenhum deles referiu dúvidas relacionadas a esse tema.

No que se refere ao consumo de bebida alcoólica, quatro pacientes (40\%) manifestaram dúvidas com relação à possibilidade de consumo de cerveja e vinho:
Olha! Fumar eu já parei desde que tive o infarto. Mas uma cervejinha no fim de semana pelo menos eu posso tomar, certo? E um vinho que às vezes costumo tomar com minha esposa, vai fazer mal? Porque eu já ouvi falar que o vinho até faz bem pro coração! (P.10).

Quatro sujeitos (40\%) referiram dúvidas quanto à continuidade dos medicamentos de uso anterior $\mathrm{e}$ adaptação aos efeitos colaterais:

Será que eu vou ter que ficar tomando remédio pro resto da vida e ficar dependente? (P.2).

[...] quais efeitos colaterais eu posso ter? A pressão pode subir? Pode disparar meu coração? (P.9).

A entrevista também permitiu identificar outras dúvidas, em cinco pacientes (50\%), relacionadas à possibilidade de frequentar festas e atividades de lazer, prevenção do IAM, exposição solar, alterações no padrão de sono e autonomia sobre si mesmo:

Aos sábados, às vezes eu gosto de ir às festas do clube que sou sócio, eu posso frequentar festas? (P.10).

Será que o sol vai fazer mal prá mim? É proibido? Em relação à cirurgia, eu vou poder ter um sono tranquilo? Ou devido à cirurgia pode mexer com alguma coisa? (P.1).

Eu vou poder ser agitada do jeito que eu era $e$ comandar minha vida? (P.9).

\section{DISCUSSÃO}

Observou-se maior número de pessoas do sexo masculino com idade média superior a 50 anos. Durante os anos que antecedem o climatério, as mulheres parecem estar protegidas contra a doença cardíaca, devido a fatores hormonais; isso pode explicar o maior índice de infarto no sexo masculino ${ }^{(12)}$.

O efeito da faixa etária sobre as patologias cardíacas, decorre do fato que essas doenças são agravadas com a idade, em função de maior tempo de exposição aos fatores de risco e que nos indivíduos idosos os mecanismos fisiopatológicos específicos que causam distúrbios nos substratos cardíacos e vasculares são modificados pelo envelhecimento ${ }^{(13)}$. 
Alguns autores afirmam que os pacientes portadores de cardiopatias referem mais sensação subjetiva de cansaço e dispnéia quando comparados a indivíduos saudáveis da mesma idade e peso, resultado do desequilíbrio da oferta e demanda de oxigênio pelo miocárdio, isso talvez possa explicar o fato da maioria da amostra ser sedentária ${ }^{(14)}$.

Com relação ao mercado de trabalho $50 \%$ dos participantes eram assalariados, fator que pode estar relacionado ao estresse devido à sobrecarga profissional e grupos de pesquisa constataram que o estresse é considerado um fator de risco reversível para a $\mathrm{DAC}^{(2)}$.

A grande maioria dos participantes referiu dúvidas quanto ao tema hábitos alimentares, pois alegou possuir dieta desequilibrada e rica em exageros. A preocupação em seguir planos de dietas alimentares evidencia a dificuldade em lidar com a reeducação de hábitos e costumes antigos, modificar a frequência e tipo de consumo de alimentos pode significar, para esses pacientes, abdicar de uma de suas maiores fontes de prazer. Estudo realizado evidenciou que 19,2\% dos pacientes que haviam realizado RVM não aderiram à dieta prescrita $^{(7)}$.

É de grande relevância que o paciente tenha consciência de que dieta desequilibrada é um fator de risco modificável para $\mathrm{DAC}^{(2)}$. O consumo de vegetais, frutas, grãos integrais, fibras alimentares e outras substâncias são de fundamental importância na prevenção e controle da DAC, assim como readequação do peso por meio do consumo moderado de gorduras e calorias ${ }^{(15)}$.

Dentre os sujeitos entrevistados, muitos se mostraram conscientes da necessidade de adquirir o hábito de realizar atividades físicas. As dúvidas mais frequentes estiveram relacionadas ao tipo, duração, frequência e momento em que poderão iniciar os mesmos.

Os sujeitos da pesquisa relacionaram o retorno ao trabalho a atividades que demandam algum tipo de esforço físico e por isso questionaram se poderiam retornar a elas ou se teriam que optar pela aposentadoria.

Em relação aos sintomas aos quais os pacientes deveriam se atentar e qual a conduta que deveriam ter após a alta, o medo da dor devido à recorrência de IAM foi a dúvida mais frequente. Dificuldades relacionadas aos sintomas de complicação após a alta são esperados considerando que o indivíduo se encontra longe dos cuidados da equipe de saúde ${ }^{(8)}$.

Destaca-se o importante papel do enfermeiro na assistência ao paciente submetido a RVM, visto que um bom histórico de enfermagem permite avaliar os conhecimentos prévios do indivíduo acerca de sua patologia. Sendo assim, quando o enfermeiro realiza o levantamento dos sintomas acaba por possuir ferramentas que o ajudam a fornecer uma melhor orientação no período da alta hospitalar, possibilitando ao sujeito uma vigilância mais rigorosa na descoberta precoce de eventuais complicações pós-operatórias.

Novamente as dúvidas dos pacientes em relação às atividades que desenvolvem diariamente estiveram relacionadas àquelas que demandam esforço físico, tais como: realizar serviços domésticos, caminhar, carregar peso, dirigir, andar de ônibus, subir escadas, entre outras. Observa-se a presença de discursos que demonstraram sentimentos de perda de controle e impotência.

Os achados desse estudo corroboram com outro, no qual após observação de pacientes em pósoperatório de cirurgia cardíaca que receberam o diagnóstico de enfermagem de impotência, destacouse entre as características definidoras expressão de dúvida acerca do desempenho de papéis e expressões de insatisfação e frustração pela inabilidade no desempenho de tarefas e/ou atividades pessoais ${ }^{(9)}$.

De todos os sujeitos entrevistados, a maioria relatou desconhecimento com relação ao tempo do processo de cicatrização das incisões cirúrgicas e permanência da cicatriz. A recuperação após esternotomia dificulta a readaptação ao cotidiano, gerando dor, irritação e até latejamento na área adjacente à cicatrização, principalmente quando os pacientes tossem, movimentam-se e desenvolvem movimentos respiratórios amplos. O desconforto ocasionado por esses fatores pode resultar em dificuldades em encontrar posição confortável para induzir ou manter o sono, repouso inadequado e dependência na realização de atividades como higiene pessoal ${ }^{(10)}$.

A preocupação com a cicatriz é constante nos pacientes que serão submetidos à cirurgia cardíaca ${ }^{(16)}$. Acredita-se que as cicatrizes deixadas pela cirurgia serão lembranças de uma etapa traumática da vida desses pacientes, além de comprometerem a aparência do indivíduo, podendo ocasionar distorção de sua autoimagem.

Outra dúvida relatada por alguns pacientes e que demonstra que o enfermeiro deve estar atento refere-se às orientações relacionadas aos cuidados de higiene com as incisões, já que em ambiente hospitalar o curativo é realizado somente pela equipe 
de enfermagem e, no momento da alta, é natural que o paciente sinta-se inseguro quanto aos cuidados que deverão ser seguidos.

Apenas quatro sujeitos alegaram possuir dúvidas no que se refere ao tempo de retorno às atividades sexuais e eventuais prejuízos e limitações no desempenho dessa atividade. Não é estranho que as dúvidas relacionadas às atividades sexuais estejam entre as menos frequentes, pois visualiza-se uma dificuldade entre os sujeitos em abordar este assunto ${ }^{(8)}$.

Ao investigar o tabagismo como fator de risco para DAC, verificou-se que todos os fumantes alegaram ter abandonado o vício após a ocorrência do IAM. É fundamental que os pacientes tenham a iniciativa de abandonar o uso de cigarros, mas é importante salientar que os mesmos encontrarão muitas dificuldades, visto que a abstinência pode desencadear irritabilidade, inquietação, piora da concentração, sintomas depressivos, alteração do sono, aumento de peso e desejo incontrolável de fumar, levando muitas pessoas a não obterem êxito na sua decisão ${ }^{(7)}$.

O profissional de saúde deve incentivar o paciente quanto ao abandono do tabagismo fornecendo orientações sobre os malefícios que esse mal pode causar e os benefícios que teriam com o fim deste vício, como o fato de que os não-fumantes vivem, em média, sete anos a mais que os fumantes e que a suspensão do fumo diminui a taxa de reinfarto e morte em cerca de um terço, em um ano(17).

Quanto ao consumo de bebidas alcoólicas, observa-se que apenas um sujeito se considerava etilista e os outros alegaram consumo esporádico. Ao serem questionados sobre o consumo de bebida alcoólica os mesmos relataram dúvidas referentes à frequência, tipo e quantidade de bebida que poderiam ingerir.

É preconizado um consumo máximo diário de álcool de uma dose (14g de álcool - uma lata de cerveja ou um copo de vinho com 120ml) para mulheres e duas doses para homens ${ }^{(15)}$. Esses dados podem auxiliar 0 profissional nas orientações fornecidas aos pacientes.

Alguns participantes manifestaram preocupações referentes à prescrição de novos medicamentos, seus possíveis efeitos colaterais e permanência de medicações que já utilizavam previamente à cirurgia. Outros questionaram a necessidade da continuidade do tratamento medicamentoso após a alta, pois relataram que se sentiam curados e que, portanto, não precisariam mais fazer uso desses remédios.

Essas preocupações também podem estar associadas ao fato de que o tratamento farmacológico representa o maior fator de custo para o tratamento das DCV. O alto custo dos medicamentos também se destaca como uma grande justificativa para a não adesão ao tratamento por parte dos pacientes ${ }^{(18)}$.

A preocupação referente às alterações no padrão de sono constitui uma das principais queixas dos pacientes em decorrência da dor, dificuldades de relaxamento, além de consequências das alterações nos ciclos de sono decorrentes das rotinas hospitalares, principalmente das unidades de terapia intensiva.

Mais uma vez ressalta-se a importância das orientações do enfermeiro no período que antecede a alta hospitalar e do posterior acompanhamento ambulatorial dos pacientes, onde dúvidas como essas devem ser esclarecidas, considerando o perfil e as características de cada indivíduo, favorecendo assim uma melhor adesão ao tratamento e prevenção de complicações.

\section{CONCLUSÃO}

O presente estudo permitiu identificar as dúvidas manifestadas por pacientes em pós operatório de RVM, sendo que as mais frequentes estiveram relacionadas, respectivamente, à reeducação dos hábitos alimentares, prática de exercícios físicos, retorno ao trabalho, sintomas vivenciados após a alta hospitalar, prática das atividades diárias, cuidados com as incisões cirúrgicas, prática de atividades sexuais, consumo de bebida alcoólica e continuidade no uso de medicações.

Constatou-se no decorrer deste estudo que inúmeras dúvidas podem surgir durante o período de reabilitação pós RVM e o conhecimento das mesmas poderá auxiliar o enfermeiro a direcionar a assistência de enfermagem, fornecendo subsídios para a elaboração de um plano de cuidados, implementação de intervenções, treinamento e qualificação da equipe de enfermagem.

Acredita-se na importância da implantação da assistência integral e individualizada, voltada às necessidades do paciente durante todas as fases de sua reabilitação. Após o conhecimento dessas dúvidas, é fundamental a realização de estudos voltados à elaboração de intervenções e métodos educativos no período que antecede a alta hospitalar. Essa estratégia poderá transmitir confiança, tranquilidade e conhecimento para que os pacientes enfrentem esse momento, prevenindo complicações e reinternações. Como o resultado pode contribuir na prática e na pesquisa em enfermagem 


\section{REFERÊNCIAS}

1. Gasperi PD, Radunz V, Prado ML. Procurando reeducar hábitos e costumes: o processo de cuidar da enfermeira no pré e pós-operatórios de cirurgia cardíaca. Cogitare Enferm. 2006 Set/Dez;11(3):252-7.

2. FHS - Framingham Heart Study. Disponível: http:// www.framinghamheartystudy.org. Acesso em: 26 abr. 2008.

3. Griffin BP, Topol EJ. Manual de medicina cardiovascular. $2^{\mathrm{a}}$ ed. Rio de Janeiro: Guanabara Koogan; 2007.

4. Benchimol CB, Carvalho LAF. Intervenção coronariana percutânea e terapêutica clínica não são adversárias no tratamento do paciente coronariopata. Rev SOCERJ. 2007 Mai/Jun;20(3):233-4.

5. Vargas TVP, Maira EM, Dantas RAS. Sentimentos de pacientes no pré-operatório de cirurgia cardíaca. Rev Latino-Am Enferm. 2006 Mai/Jun;14(3):383-8.

6. Lobiondo-Wood G, Haber J. Pesquisa em enfermagem: métodos, avaliação crítica e utilização. Rio de Janeiro: Guanabara Koogan; 2001.

7. Lima FET, Araújo TL. Prática do autocuidado essencial após a revascularização do miocárdio. Rev Gaúcha Enferm. 2007;28(2):223-32.

8. Dantas RAS, Aguillar OM. Problemas na recuperação de pacientes submetidos à cirurgia de revascularização do miocárdio: o acompanhamento pelo enfermeiro durante o primeiro mês após a alta hospitalar. Rev Latino-Am Enferm. 2001 Nov/Dez;9(6):31-6.

9. Braga CG, Cruz DAELM. A resposta psicossocial de impotência em pacientes no pós-operatório de cirurgia cardíaca. Rev Esc Enferm USP. 2003 Mar;37(1):26-35.

10. Dutra CMP, Coelho MJ. O tempo de cicatrização do coração: cuidar e os cuidados do cliente para superar as dificuldades após cirurgia cardíaca. Enfermería Global. 2008 Fev;2:1-12.

11. Simioni AMC. O gerenciamento de recursos humanos em saúde como processo social [dissertação]. São Paulo (SP): Universidade de São Paulo; 1997.

12. Meltzer LE. Enfermagem na unidade coronariana. São Paulo: Atheneu; 1997.

13. Smeltzer SC, Bare BG. Tratado de enfermagem médicocirúrgica. 10ªed. Rio de Janeiro: Guanabara Koogan; 2005.
14. Moraes RS, editor. Diretriz de reabilitação cardíaca. Arq Bras Cardiol. 2005 Nov/Dez;84(5):431-40.

15. American Heart Association (AHA). Scientific Statement: Summary of the scientific conference on dietary fatty acids and cardiovascular health. Conference summary from the nutrition committee of The American Heart Association. Circulation. 2001;103:1034-9.

16. Souza RHS. Sentimentos e percepções do cliente no pré-operatório de cirurgia cardíaca [dissertação]. Curitiba (PR): Universidade Federal do Paraná; 2004.

17. Piegas LS, editor. III Diretriz sobre tratamento do infarto agudo do miocárdio. Arq Bras Cardiol. 2004 Set;83(4)(supl.4):1-86.

18. Ribeiro RA, Mello RGB, Melchior R, Dill JC, Hohmann $\mathrm{CB}$, Lucchese AM, et al. Custo anual do manejo da cardiopatia isquêmica crônica no Brasil. Perspectiva pública e privada. Arq Bras Cardiol. 2005 Jul;85(1): 3-8. 